\section{Comments on "Recognition of faces in the presence of two-dimensional sinusoidal masks" by Tieger and Ganz}

\author{
DAVID RILEY and ALAN COSTALL \\ University of Southampton, Southampton, England
}

The recent paper by Tieger and Ganz (1979) on the effects of sinusoidal masks on the recognition of faces concludes that spatial frequencies around 2.2 cycles/deg visual angle "appear to be relatively more important than other frequencies in the face recognition task" (p. 165). Their experimental paradigm was to superimpose on a picture of a face, a two-dimensional sinusoidal grid of discrete spatial frequency of $.54, .82,1.1,2.2$, or 3.9 cycles $/ \mathrm{deg}$. They found a very clear peak in the masking function at $2.2 \mathrm{cycles} / \mathrm{deg}$, despite the fact that no such peak occurs in the human modulation transfer function or in the energy spectra of the photographs they employed. Although they close their paper with a brief note of scepticism concerning the value of the linear systems approach, they explain their results in terms of the existence of a secondary, attentional filter which results in the maximal weighting of spatial frequencies within this middle range.

We would like to reconsider the interpretation of the effect of their grid mask on face recognition. We are not questioning their finding of a greater disruptive effect for the 2.2 -cycle/deg mask; their results are supported by Woodhouse (1976) and by ourselves, using low-pass filtering techniques which reveal the existence of useful information in the lower spatial frequencies of the face.

The problem with their account is that it fails to consider the fact that faces are encountered and recognized at distances other than the one employed in their experiment. Their conclusion that the important spatial frequency constituents of the face lie around 2.2 cycles/deg needs some kind of qualification, namely, that it holds only for the particular viewing conditions of their experiment. We are making the obvious point that these spatial frequencies cannot be the critical ones for face recognition in general.

Tieger and Ganz propose that their attentional filter peaks at around 2.2 cycles/deg because the internal features of the face in their experiment lie within this region of the frequency spectrum. We would like to point out that the spatial frequency

David Riley would like to acknowledge the financial support of the Social Science Research Council. Authors' address: Department of Psychology, The University, Southampton, SO9 5NH, England. components of the face will vary with viewing distance, and so the weight of this selective filter would need to alter for each and every viewing position. The filter would need to sweep up and down the frequency spectrum to be of much use to anyone outside the confines of their experiment.

An alternative approach, which they suggest but do not pursue, is in terms of the spatial stimulus domain rather than the Fourier domain, that is, in terms of the salient features of the face. There is certainly evidence for the relative importance of the internal features in face recognition (Ellis, Shepherd, \& Davies, 1979), but it is not clear how Tieger and Ganz obtained their estimate of 2 cycles/deg for the corresponding spatial frequency, given the large discrepancy between the $\mathrm{X}$ and $\mathrm{Y}$ dimensions of these features (i.e., an absence of radial symmetry). In any case, a simple demonstration based on the example of the stimulus material (Figure 1 in their paper) shows that the useful information for recognition must lie below 2.2 cycles/deg. If the photograph of the simulated stimulus with grid mask of 2.2 cycles/ deg is blurred, low-pass filtered, or viewed from a distance of 3 to $4 \mathrm{~m}$, the internal features of the face are easier to perceive.

It is tempting to conclude, therefore, that the really useful information for face perception lies between 1.1 and $2.2 \mathrm{cycles} / \mathrm{deg}$ (the effect of the 2.2 cycles/ deg mask being explained on the basis of critical band masking). Our point, however, is that such a representation of the results would be misleading, in view of the considerable radial asymmetry of the features of the face. Given the structure of the face, it is clear that quite different results would have been obtained if the masks had been obliquely oriented, or if a peak rather than a trough of the sinusoid happened to superimpose the eyes, for example, of the displayed face. ${ }^{1}$ Furthermore, there is increasing evidence for a variety of strategies and information which may be used to identify faces (Mathews, 1978; Walker-Smith, Gale, \& Findlay, 1977). This presents problems for a model which proposes any one source of information or any one strategy as being responsible for face recognition. In brief, we are suggesting that despite the promises of fresh insights arising from the linear systems approach, the Fourier description itself may only be comprehensible in the light of what we already know about the human face.

\section{REFERENCES}

Ellis, H. D., Shepherd, J. W., \& Davies, G. M. Identification of familiar and unfamiliar faces from internal and external features: Some implications for theories of face recognition. Perception, 1979, 8, 431-439. 
HARMON, L. D. Some aspects of the recognition of human faces. In O.-J. Grusser \& R. Klinke (Eds.), Pattern recognition in biological and technical systems. New York: Springer-Verlag, 1971.

Matuews, M. L. Discrimination of Identikit constructions of faces: Evidence for a dual processing strategy. Perception \& Psychophysics, 1978, 23, 153-161.

Tifger, T., \& Ganz, L. Recognition of faces in the presence of two-dimensional sinusoidal masks. Perception \& Psychophysics, 1979, 26, 163-167.

Walker-Smith, G. J., Gale, A., \& Findlay, J. M. Eye movement strategies involved in face perception. Perception, 1977, 6, 313-326.

WoODHOUSE, J. M. The application of contrast sensitivity to studies on image detection and recognition. Unpublished doctoral dissertation, University of Cambridge, 1976.
NOTE

1. A similar possibility is suggested by Harmon (1971, p. 212). He produced degraded facial images which, when viewed close up, look like cubistic paintings. He notes that the position of the individual blocks composing the picture may land fortuitously or unluckily for adequate representation.

(Received for publication November 19, 1979; accepted January 9,1980 .) 